\title{
Predicting postoperative complications after bariatric surgery: the Bariatric Surgery Index for Complications, BASIC
}

\author{
Usha K. Coblijn ${ }^{1}$ - Julian Karres ${ }^{2}$. Christel A. L. de Raaff ${ }^{1} \cdot$ Steve M. M. de Castro $^{3}$. \\ Sjoerd M. Lagarde ${ }^{4} \cdot$ Willem F. van Tets ${ }^{1} \cdot$ H. Jaap Bonjer ${ }^{5} \cdot$ Bart A. van Wagensveld $^{1}$
}

Received: 30 July 2016 / Accepted: 24 February 2017 / Published online: 31 March 2017

(C) The Author(s) 2017. This article is an open access publication

\begin{abstract}
Background Around 20\% of bariatric surgery patients develop a short- or long-term complication.

Objective Aim of this study was to develop a risk model predicting complications: the Bariatric Surgery Index for Complications (BASIC).

Setting The Obesity Center Amsterdam, located in a large teaching hospital, in Amsterdam, The Netherlands.

Methods A prospective consecutive database including patients operated between November 2007 and February 2015 was used. For the BASIC, analysis according to the TRIPOD statement was performed to identify risk factors for complications. Class I included patients with zero to one risk factor, class II patients with two risk factors, and class III patients with three or more risk factors.

Results Of 1709 analyzed patients, mean age was 45 years $( \pm$ SD 10.7$), 1393(81.5 \%)$ were female; mean body mass index was $44.5 \mathrm{~kg} / \mathrm{m}^{2}$ (6.8). Overall, 271 (15.9\%) patients developed a complication of which 197 (72.5\%) occurred within 30 days. Predictors in multivariable analysis were
\end{abstract}

Usha K. Coblijn

usha.coblijn@gmail.com

1 Departments of Surgery, Onze Lieve Vrouwe Gasthuis, locatie West (Prevoiusly Sint Lucas Andreas Ziekenhuis, Amsterdam), Jan Tooropstraat 161, 1064 AE Amsterdam, The Netherlands

2 Departments of Surgery, Tergooi Ziekenhuizen, Blaricum, The Netherlands

3 Departments of Surgery, Onze Lieve Vrouwe Gasthuis, Amsterdam, The Netherlands

4 Departments of Surgery, Erasmus Medisch Centrum, Rotterdam, The Netherlands

5 Departments of Surgery, Vrije Universiteit Medisch Centrum, Amsterdam, The Netherlands use of anticoagulants (odd's ratio (OR) 1.5); chronic obstructive pulmonary disease (OR 2.3); dyslipidemia (OR 1.4); gender (OR 1.4); psychiatric history (OR 1.3); and revisional surgery (OR 1.5). In class I, 13.5\% (181 out of 1338) experienced complications, in class II 58 (21.6\%) of the 269 patients and in class III $32(31.4 \%)$ of the 102 patients, respectively. There was a significant difference $(p<0.001)$ in both overall and 30 day complications.

Conclusion The BASIC uses six preoperative variables to classify patients in a low-, intermediate-, or high-risk group for postoperative complications after bariatric surgery.

Keywords Bariatric surgery - Complications - Prediction model $\cdot$ Roux-en-Y gastric bypass $\cdot$ Sleeve gastrectomy

Obesity is a major health problem and the incidence is increasing worldwide. So far, the only treatment for morbid obesity with good long-term results is bariatric surgery. Recently, there is a shift from laparoscopic adjustable gastric banding (LAGB) to laparoscopic Roux-en Y gastric bypass (LRYGB) and laparoscopic sleeve gastrectomy (LSG). At present, about $49 \%$ of procedures are LRYGB [1]. This procedure has become relatively safe with acceptable morbidity and mortality [2,3].

As a predictive tool for postoperative mortality, the obesity surgery mortality risk score (OS-MRS) can be used [4, 5]. This score consists of the following parameters: body mass index $(\mathrm{BMI}) \geq 50 \mathrm{~kg} / \mathrm{m}^{2}$; age $\geq 45$; male gender; hypertension; risk of pulmonary embolism. Although it is unknown if the OS-MRS accurately predicts the risk for postoperative complications, it is sometimes used for that purpose [6,7].

A common method to inventory postoperative complications is the Clavien-Dindo classification [8], which is based 
on the required intervention following complications and ranges from no intervention, reoperation, or radiological intervention, intensive care unit admission, and death. After laparoscopic bariatric surgery, the incidence and severity of short-term (within 30 days postoperatively) adverse events vary between the 4.9 and 10\% [9-11]. Severe complications such as anastomotic/ staple line leakage, stenosis or stricture of the anastomosis and pulmonary embolism occur in $3 \%$ of the patients; death in $0.2 \%$ [6, 12]. Preoperative risk assessment is one of the most important components of surgical decision making. A risk assessment system for bariatric surgery should provide an accurate representation of the complication risk using only the information that is preoperatively available The aim of this study is to develop the Bariatric Surgery Index for Complications (BASIC), a simple, adequate scoring system, similar to the OS-MRS based on preoperative parameters, assessing the risk on postoperative complications.

\section{Methods}

\section{Study cohort}

All patients who underwent primary or revisional LRYGB or LSG or pouch revision of previous LRYGB from November 2007 onwards were prospectively entered in a consecutive database. Patients operated until January 2014 were included in the analysis, as they had a minimal follow-up of 12 months. Patients were selected according to their surgical procedure. Included were those who require a staple line or anastomosis as all these kind of surgeries contain the risk of anastomotic or staple line leakage. Patients who underwent laparoscopic gastric banding or banded bypass as secondary stage procedure were excluded from analysis. Patients eligibility regarding bariatric surgery was screened according to the IFSO guidelines [13].

All patients at our center receive a routine preoperative screening by a multidisciplinary team including a medical doctor, prior to surgery. All patients are interviewed concerning their medical history and drug usage, a full physical examination is performed and no patient is operated without information of their general practitioner. If the suspicion of any disease (e.g., diabetes, dyslipidemia) or insufficient treatment of existing disease exist patients are subjected to the appropriate diagnostics before they can proceed to surgery. Screening for obstructive sleep apnea (by poly(somno)graphy), hypertension, the presence of $\mathrm{H}$. Pylori [with oesophagogastroduodenoscopy and CLO test (prior to July 2014) or feces test (after July 2014)] and psychopathology by means of a consult with a psychotherapist and usage of questionnaires is mandatory in all patients prior to surgery.
The required data for this risk prediction model were collected from the database. Patient characteristics, such as age, gender, comorbidities, BMI, and operative data were retrieved. Complications were scored by both type and severity using the Clavien-Dindo classification with the following endpoints: mortality; multi-organ failure; single organ failure; surgical intervention; radiological intervention; medical treatment; no intervention needed; and no complications. A Clavien-Dindo classification of three points or higher was regarded as a severe complication. The investigators, collecting the data for the database, based on the electronic patient status, assigned the Clavien-Dindo classification points. In addition to this classification, the nature of the complication was scored as well as if it were short-term (occurring within 30 days after surgery) or longterm complications. A complication graded Clavien-Dindo 3 or higher was considered a serious complication, those were also separately analyzed in the risk stratification.

This study has been approved by the local medical ethics committee; no individual informed consent was necessary as it was a retrospective analysis.

Twenty-four patient variables within the database were regarded as possible risk factors and subsequently analyzed for postoperative complications (Appendix Table 8). COPD was defined as being diagnosed by a pulmonary doctor with at least COPD GOLD II; diabetes was divided in patients with and without insulin usage; dyslipidemia was scored when patients used cholesterol lowering drugs or were diagnosed with dyslipidemia by means of a blood test; OSA was diagnosed in every patient by means of a poly(somno) graphy; a psychiatric disease was diagnosed when patients used psychiatric drugs (including anti-depressants) and/or went through extensive therapy; all gastric disorders were found by the performance of a gastroscopy preoperatively; the usage of NSAID's and corticosteroids were based on medication history taking and finally the usage of anticoagulants was defined as the chronic usage of any anticoagulant including platelet inhibitors. Variables with more than 5\% missing data were discarded after sub-analysis showed no effect of these variables. For the prediction of complications, three categories were distinguished with a uni- and multivariable regression analysis: class I included patients who had a maximum of one risk factor, class II comprised patients with two risk factors and class III was with patients with three or more risk factors.

\section{Surgical procedure}

All procedures were carried out by four experienced bariatric surgeons or under their direct supervision. The procedures were performed as previous described [14]. If patients underwent a revisional procedure from an adjustable gastric band, it started with removal of the band 
followed by direct revision, after which the Port-a-cath was removed prior to skin closure.

For all procedures pneumoperitoneum was obtained. Five trocars (three $12 \mathrm{~mm}$ and two $5 \mathrm{~mm}$ ) were used. In case of LRYGB, the pouch was formed with one horizontal and 3-4 vertical firings of a $45 \mathrm{~mm}$ endoscopic stapler (Johnson and Johnson, Sommerville, NY, USA) in the lesser curvature of the stomach. Subsequently the gastrojejunostomy was created in an antecolic, antegastric fashion, posterior by means of a stapler and anteriorly hand sewn using a barbed suture V-loc ${ }^{\mathrm{TM}}$ (Covidien, Dublin, Ireland). This was followed by the jejunojejunostomy at $120-150 \mathrm{~cm}$ and transection of the connecting loop.

The LSG was created using multiple firings of the Echelon 60 endoscopic stapler. The remnant stomach was removed through the most lateral $12 \mathrm{~mm}$ port after the trocar was removed and the incision enlarged $(2-3 \mathrm{~cm})$ and sent for pathologic examination.

Pouch revision was performed after inspection in the same fashion as creation of the pouch at primary LRYGB. Often the anastomosis was revised.

\section{Statistical analysis}

All data were analyzed using SPSS version 21.0 for windows (SPSS Chicago, IL, USA).

For the BASIC, uni- and multivariable regression analyses were performed to identify the variables predicting complications. Multivariable regression analysis was performed according to the TRIPOD statement, which accepts $p$ values up to 0.157 to enhance the applicability of the model to other patient groups [15]. After classifying the patients, the Chi-square test was used to demonstrate any statistical significance, of which definition was set at a two sided $p$ value of $<0.05$. Select cases was used to detect the highest association between groups after which correction for multiple testing took place.

\section{Results}

From November 2007 till February 2015, a total of 1709 patients underwent bariatric surgery.

Most patients, 1393 (81.5\%) were female; the mean age was 44.6 years (SD 10.7) and the mean BMI was $44.5 \mathrm{~kg}$ / $\mathrm{m}^{2}$ (SD 6.8). Baseline characteristics are displayed in Table 1.

Primary LRYGB was performed in 1283 patients (75.1\%), followed by revision from LAGB into LRYGB in 281 patients (16.4\%) (Table 2).

Overall, postoperative complications occurred in 271 patients (15.9\%) of which $197(72.7 \%)$ were short-term complications, (Table 3). Twenty-two patients had a
Table 1 Baseline characteristics

\begin{tabular}{llll}
\hline Baseline & No complication & Complication & $p$ value \\
\hline Age (years) (SD) & $44.4(10.7)$ & $45.9(10.7)$ & $\mathbf{0 . 0 3 5}$ \\
BMI (kg/m ${ }^{2}$ ) (SD) & $44.6(6.7)$ & $43.8(7.1)$ & 0.097 \\
Gender (F/M) & $1183 / 255$ & $210 / 61$ & 0.063 \\
Diabetes (\%) & $403(28.0)$ & $87(32.1)$ & 0.175 \\
Dyslipidemia (\%) & $317(22.1)$ & $81(30.1)$ & $\mathbf{0 . 0 0 4}$ \\
Hypertension (\%) & $572(39.8)$ & $130(48.0)$ & $\mathbf{0 . 0 1 2}$ \\
Revisional surgery (\%) & $249(17.3)$ & $68(25.1)$ & $\mathbf{0 . 0 0 3}$ \\
\hline
\end{tabular}

Bold values indicate statistical analysis at $p<0.05$

$B M I$ body mass index, $F$ female, $M$ male

leakage of the gastrojejunostomy (GJS), 42 patients suffered from (severe) peri- or postoperative bleeding, 15 patients had a stenosis of the GJS, and ten patients developed an internal herniation approximately 1 year after surgery. Of all 271 complications, 140 patients (8.2\% of 1709) had a severe complication according to the Clavien-Dindo classification. Five patients (0.3\%) died (Clavien-Dindo 5), three of them had revisional surgery and subsequently died of cardiac tamponade, pulmonary embolism, and bowel strangulation, respectively, two patients died of sepsis and multi-organ failure after anastomotic leakage from primary LRYGB.

\section{Risk analysis}

Twenty-four preoperative variables were considered in the univariate analysis as a risk predictor whereof five were significant $p<0.05$ : age of 60 years and above; hypertension; dyslipidemia; chronic obstructive pulmonary disease (COPD); and revision from previous bariatric surgery. Use of anticoagulants and a history of psychiatric diseases were added according to the TRIPOD statement. In the multivariable analysis, backwards selection resulted in elimination of diabetes type II; followed by age above 60; alcohol; corticosteroids; BMI above 50; gastroesophageal reflux disease; smoking; NSAID's; cholecystectomy; hypertension and history of trombo-embolic event respectively. Consequently, anticoagulant usage; COPD; dyslipidemia; gender; psychiatric history, and revisional surgery provided the most optimal multivariable model as displayed in Table 4. As all factors had an odd's ratio between the 1.3 and 2.3, one point was assigned to each of the contributing factors.

\section{Risk classification}

Patients were divided in classes using the variables according to the multivariable analysis and the description in the "methods" section. A differentiation was made between short-term and overall complications. The overall 
Table 2 Type of surgery

\begin{tabular}{lrl}
\hline Procedure & Total number & Percentage \\
\hline LRYGB & 1283 & 75.1 \\
LSG & 109 & 6.4 \\
LSG to LRYGB & 15 & 0.9 \\
LAGB to LRYGB & 281 & 16.4 \\
LAGB to LSG & 11 & 0.6 \\
Mason to LRYGB & 1 & 0.1 \\
Mason to LSG & 1 & 0.1 \\
Pouch revision & 8 & 0.5 \\
Total procedures & 1709 & 100 \\
\hline
\end{tabular}

$L R Y G B$ laparoscopic Roux en Y gastric bypass, $L S G$ laparoscopic sleeve gastrectomy $L A G B$ laparoscopic adjustable gastric band

Table 3 Number of complications

\begin{tabular}{llc}
\hline Complication & Total number & Percentage \\
\hline Short-term & 197 & 11.5 \\
Overall & 271 & 15.9 \\
Clavien-Dindo $\geq 3$ & 140 & 8.2 \\
\hline
\end{tabular}

Table 4 Multivariable analysis, risk factors BASIC

\begin{tabular}{lllll}
\hline Variable & $p$ value & Odd's ratio & \multicolumn{2}{c}{$\begin{array}{l}\text { 95\% CI for the } \\
\text { Exp. }\end{array}$} \\
\hline Anticoagulants & 0.142 & 1.454 & 0.883 & 2.394 \\
COPD & 0.007 & 2.271 & 1.254 & 4.113 \\
Dyslipidemia & 0.042 & 1.396 & 1.012 & 1.928 \\
Gender (male) & 0.037 & 1.438 & 1.023 & 2.023 \\
Psychiatric history & 0.137 & 1.298 & 0.921 & 1.831 \\
Revisional surgery & 0.021 & 1.498 & 1.064 & 2.110 \\
\hline
\end{tabular}

$C O P D$ chronic obstructive pulmonary disease

complication analysis showed the following results: class I existed of 1338 (78.3\%) patients of which 181 (13.5\%) suffered from a complication, class II comprised 269 patients (15.7\%) of which $142(21.6 \%)$ patients had a complication and class III existed of 102 patients $(6.0 \%)$ of which 32 (31.4\%) developed a complication. The difference in incidence of complications between the three classes was statistically significant with a $p$ value of 0.001 (Table 5).

Not only was this significant in the occurrence of overall complications but also within the patient group who developed a short-term complication $(p=0.001)$. These shortterm complications occurred in $136(10.2 \%)$ of the patients in class I, 40 (14.9\%) patients in class II, and 21 (20.6\%) patients in class III, respectively (Table 6).

The significant difference between classes was mainly caused by the difference between class I and III but the
Table 5 Distribution among classes over all complications

\begin{tabular}{llll}
\hline Classification & Total number (\%) & Complication (\%) & $p$ value \\
\hline $\begin{array}{l}\text { Class I (0-1 risk } \\
\text { factor) }\end{array}$ & $1338(78.3)$ & $181(13.5)$ & $\mathbf{0 . 0 0 1}$ \\
$\begin{array}{l}\text { Class II (2 risk fac- } \\
\text { tors) }\end{array}$ & $269(15.7)$ & $58(21.6)$ & \\
$\begin{array}{l}\text { Class III ( } \geq 3 \text { risk } \\
\text { factors) }\end{array}$ & $102(6.0)$ & $32(31.4)$ & \\
\hline
\end{tabular}

Bold value indicates statistical analysis at $p<0.05$

difference between all classes was significant in the overall complication rate. Analyzing short-term complications alone, the significance was mainly caused by comparison of class I and III, followed by I and II. No difference between II and III could be identified. When dividing complications in mild (Clavien-Dindo $\leq 3$ ) and severe (Clavien-Dindo $>3$ ) complications, a significant difference exists between class I versus III and class I versus II.

The results of this sub-analysis are displayed in Table 7.

\section{Discussion}

This study developed a risk model for postoperative complications in an attempt to predict the development of complications after bariatric surgery. The study identified six preoperative variables, which are all independent risk factors for the occurrence of postoperative complications. With this risk model, it is possible to select patients with a two times higher risk of postoperative complications compared to the general bariatric population.

It might seem odd that dyslipidemia was identified as a risk factor for postoperative complications. However, as one of the pillars of metabolic syndrome, patients with dyslipidemia are in less good condition or shape than patients without dyslipidemia. Furthermore the presence of dyslipidemia increases the likelihood of cardiovascular diseases and maybe also cardiovascular complications. For example, ischemia is thought to be a part in the pathophysiology of the development of anastomotic leakage after bariatric surgery or in marginal ulceration.

Numerous authors have attempted to predict the risk for complications after bariatric surgery. Some by adjusting or applying the OS-MRS to their population $[6,16]$, others by developing a new model based on national databases [17-19]. Gupta et al. provided a risk prediction model that calculates the risk of major postoperative complications. The model was based on the following variables; type of surgery, extremes of BMI 35 to $<45$ and $>60 \mathrm{~kg} /$ $\mathrm{m}^{2}$, recent myocardial infarction, bleeding disorder, functional dependency in daily life, hypertension, and stroke. The complications were divided into 17 possibilities 
Table 6 Distribution among classes short-term complications

\begin{tabular}{llll}
\hline Classification & Total number (\%) & Complication (\%) & $p$ value \\
\hline $\begin{array}{l}\text { Class I (0-1 risk } \\
\text { factor) }\end{array}$ & $1338(78.3)$ & $136(10.2)$ & $\mathbf{0 . 0 0 1}$ \\
$\begin{array}{l}\text { Class II ( } 2 \text { risk fac- } \\
\text { tors) }\end{array}$ & $269(15.7)$ & $40(14.9)$ & \\
$\begin{array}{l}\text { Class III ( } \geq 3 \text { risk } \\
\text { factors) }\end{array}$ & $102(6.0)$ & $21(20.6)$ & \\
\hline
\end{tabular}

Bold value indicates statistical analysis at $p<0.05$

according to their nature or required intervention $[12,17]$. BMI was also analyzed in the present study, as continuous and dichotomous variable but was not associated with complications. This might be due to the increased experience in large volume centers causing less influence of BMI on operative outcome. Therefore, concentrating bariatric surgery in large volume centers might be important to improve the results. Subsequently, Birkmeyer et al. found that surgical skill was strongly related to volume and not to clinical important differences as patient age, sex, or BMI. Obviously, they found a difference in surgical skill and complication rate; however, the present research was performed in one high-volume center, automatically eliminating the bias of different centers or surgeons who perform less procedures annually [20]. Due to increasing experience in bariatric procedures and high-volume centers, some previously described patient characteristics such as BMI will become less important causes in the development of postoperative complications, as demonstrated in the present study.

In contrast, functional dependency and a history of stroke were not separate variables in the present study and therefore not taken into consideration, which might be of additional value in validating this cohort or should be taken into account in future studies [12].
Although it is important to detect patients with an increased risk of severe long-term complication, it is difficult to predict this risk based on preoperative variables. Preoperative patient characteristics change as this is the primary focus of bariatric surgery with the main interest in decreasing patient's comorbidities [2,21].

One limitation of the present study is the relatively small sample size $(n=1709)$. Due to the low complication rate, detecting inter patient variability requires larger study populations.

Another limitation, partially caused by the same problem of a small sample size, is that this risk model predicts the risk on overall complications; however, it does not provide the risk factors for each complication on itself. For example, it is known is that smoking, NSAIDS, and corticosteroids increase the risk on marginal ulceration; however, these parameters did not influence the overall complication rate, possibly due to the fact that the influence of marginal ulceration in the total complication rate is small. Revisional surgery, as in the present cohort, increases the risk on postoperative complications by itself $[9,19]$. It is advisable to focus in future, prospective studies, on the differences between patients in primary and revisional bariatric surgery, such as the possibility of malnutrition. Another, possible limitation is that the parameters collected in the database were determined prior to this study; all data available on the patients were entered preoperatively and all other perioperative and postoperative data were prospectively collected. This might induce the possibility that certain variables were not collected, which would influence the risk on complications. However, the objective of this study was to identify risk factors based on preoperative patient characteristics in an attempt to select patients with a higher risk preoperatively and if possible adjust the perioperative care for which this study was sufficient.

Table 7 Select cases between groups

\begin{tabular}{|c|c|c|c|c|}
\hline BASIC & Complication rate & $p$ value & OR & CI \\
\hline \multicolumn{5}{|c|}{ Short-term complications } \\
\hline I vs. III & 10.2 vs. 20.6 & 0.001 & 2.291 & $1.374-3.822$ \\
\hline I vs. II & 10.2 vs. 14.9 & 0.024 & 1.544 & $0.056-2.257$ \\
\hline II vs. III & 14.9 vs. 20.6 & 0.185 & 1.484 & $0.826-2.666$ \\
\hline \multicolumn{5}{|c|}{ Overall complications } \\
\hline I vs. III & 13.5 vs. 31.4 & $<0.001$ & 2.922 & $1.870-4.567$ \\
\hline I vs. II & 13.5 vs. 21.6 & 0.001 & 1.757 & $1.263-2.444$ \\
\hline II vs. III & 21.6 vs. 31.4 & 0.049 & 1.663 & $0.999-2.767$ \\
\hline \multicolumn{5}{|c|}{ Severe complications } \\
\hline I vs. III & 7.2 vs. 15.3 & 0.004 & 2.330 & $1.292-4.202$ \\
\hline I vs. II & 7.2 vs. 13.0 & 0.002 & 1.931 & $1.270-2.935$ \\
\hline II vs. III & 13.0 vs. 15.3 & 0.575 & 1.207 & $0.625-2.329$ \\
\hline
\end{tabular}

Bold values indicate statistical analysis at $p<0.05$ 
Finally, all variables predicting complications in the present cohort were equally treated in the present paper despite small differences in odd's ratio's to increase the usability of this BASIC prediction model in daily clinical practice.

Validation of this risk model in a larger cohort is necessary. As many predictors were assessed, it might be possible, although highly unlikely, that the present findings are a coincidence and an expression of the general poor health of these subjects analyzed as a cohort, but no significant risk factor in the individual patient. Moreover, validation of this model in a different cohort than its development is preferable since this increases the applicability of the model in other patient groups. The development of a prediction model based on a sufficient area under the curve with good calibration is preferred but not possible in this relatively small cohort. Larger cohort studies would provide the possibility to develop a prediction model with predictive property and more detailed discrimination between patients, possible thereby enhancing the general applicability of the model.

As a final remark, it is possible, even likely, that other risk factors, are not analyzed in this study, exist and thereby influence the patient outcomes.

Although laparoscopic bariatric surgery has a low complication rate, it is performed in a patient population with significant co-morbidity, even for elective surgery. Therefore, it is important to identify patients based on their own characteristics who have a high risk on postoperative complications. As bariatric centers become more high volume, care is increasingly adjusted to accommodate the bariatric surgery patients, therefore the intercenter variability will become less a confounder in predicting complications between those centers.

\section{Conclusion}

The BASIC is based on six preoperative patient characteristics to classify patients in three risk classes: low-, intermediate-, and high-risk as class I, II, and III, respectively. This model provides the possibility to identify a small subgroup of patients with a two times higher (30.6\%) risk of overall postoperative complications following bariatric surgery.

While as of now the BASIC lacks validation, the question can be raised if patients in class III with three or more risk factors should be selected for surgery according to the same eligibility criteria or treated perioperatively under the same conditions as patients from class I or II. Preoperative risk assessment can facilitate patient specific, adjusted care and lead to improved patient outcomes after bariatric surgery.

\section{Compliance with ethical standards}

Disclosure Dr. H.J. Bonjer reports board membership of the EAES; consultancy payment form Olympus, Ethicon Endo Surgery; Expert testimony for insurance companies; Grants from ZON-MW, Ethicon and Medtronic, payment for key note lectures; Royalties from Springer. All these do not create a conflict of interest. Usha K. Coblijn; Julian Karres; Christel A.L. de Raaff; Steve M.M. de Castro; Sjoerd M. Lagarde; Willem F. van Tets and Bart A. van Wagensveld do not have a conflict of interest or financial ties to disclose.

Ethical approval For this type of study formal consent was not required and the study was approved by the local medical ethics committee.

Informed consent For this type of study, informed consent was not required. No identifying details are presented.

Open Access This article is distributed under the terms of the Creative Commons Attribution 4.0 International License (http:// creativecommons.org/licenses/by/4.0/), which permits unrestricted use, distribution, and reproduction in any medium, provided you give appropriate credit to the original author(s) and the source, provide a link to the Creative Commons license, and indicate if changes were made.

\section{Appendix}

See Table 8.

Table 8 Overall complications, univariate analysis

\begin{tabular}{|c|c|c|c|c|c|c|}
\hline Variable & $\begin{array}{l}\text { Total no of } \\
\text { patients }\end{array}$ & No of patients & Complication & $p$ value & CI $(95 \%)$ & \\
\hline \multicolumn{7}{|l|}{ Age } \\
\hline$\geq 60$ & 1709 & 155 & 30 & 0.214 & 0.857 & 1.991 \\
\hline$<60$ & & 1553 & 125 & & & \\
\hline \multicolumn{7}{|l|}{ Gender } \\
\hline $\mathrm{F}$ & 1709 & 1393 & 210 & 0.064 & 0.9833 & 1.848 \\
\hline M & & 316 & 61 & & & \\
\hline \multicolumn{7}{|l|}{ BMI } \\
\hline$\geq 50$ & 1707 & 337 & 48 & 0.360 & 0.610 & 1.197 \\
\hline$<50$ & & 1370 & 289 & & & \\
\hline
\end{tabular}


Table 8 (continued)

\begin{tabular}{|c|c|c|c|c|c|c|}
\hline Variable & $\begin{array}{l}\text { Total no of } \\
\text { patients }\end{array}$ & No of patients & Complication & $p$ value & CI (95\%) & \\
\hline \multicolumn{7}{|l|}{$\overline{\mathrm{COPD}}$} \\
\hline Yes & 1709 & 63 & 18 & 0.006 & 1.255 & 3.866 \\
\hline No & & 1646 & 45 & & & \\
\hline \multicolumn{7}{|l|}{ Diabetes } \\
\hline Yes & 1708 & 490 & 87 & 0.678 & 0.774 & 1.482 \\
\hline No & & 1281 & 403 & & & \\
\hline \multicolumn{7}{|c|}{ NIDDM } \\
\hline Yes & 1708 & 288 & 51 & 0.349 & 0.840 & 1.641 \\
\hline No & & 1420 & 237 & & & \\
\hline \multicolumn{7}{|l|}{ IDDM } \\
\hline Yes & 1708 & 209 & 37 & 0.438 & 0.794 & 1.703 \\
\hline No & & 1499 & 172 & & & \\
\hline \multicolumn{7}{|c|}{ Dyslipidemia } \\
\hline Yes & 1706 & 398 & 81 & 0.004 & 1.140 & 2.032 \\
\hline No & & 1308 & 317 & & & \\
\hline \multicolumn{7}{|c|}{ Hypertension } \\
\hline Yes & 1709 & 1007 & 130 & 0.012 & 1.076 & 1.812 \\
\hline No & & 702 & 572 & & & \\
\hline \multicolumn{7}{|c|}{ OSA Y/N } \\
\hline Yes & 1538 & 597 & 146 & 0.904 & 0.741 & 1.303 \\
\hline No & & 941 & 795 & & & \\
\hline \multicolumn{7}{|c|}{ Sever OSAS $(\mathrm{AHI}>30)$} \\
\hline Yes & 1529 & 305 & 51 & 0.542 & 0.792 & 1.559 \\
\hline No & & 1221 & 257 & & & \\
\hline \multicolumn{7}{|c|}{ Psychiatric disease } \\
\hline Yes & 1708 & 316 & 58 & 0.182 & 0.903 & 1.721 \\
\hline No & & 1391 & 258 & & & \\
\hline \multicolumn{7}{|c|}{ Trombo-embolic event } \\
\hline Yes & 1709 & 58 & 8 & 0.664 & 0.396 & 1.802 \\
\hline No & & 1651 & 50 & & & \\
\hline \multicolumn{7}{|l|}{ GERD } \\
\hline Yes & 1636 & 554 & 92 & 0.507 & 0.832 & 1.451 \\
\hline No & & 1082 & 462 & & & \\
\hline \multicolumn{7}{|l|}{ Gastritis } \\
\hline Yes & 1135 & 217 & 39 & 0.483 & 0.779 & 1.695 \\
\hline No & & 918 & 178 & & & \\
\hline \multicolumn{7}{|c|}{ Hiatal Hernia } \\
\hline Yes & 1140 & 210 & 31 & 0.500 & 0.570 & 1.316 \\
\hline No & & 930 & 179 & & & \\
\hline \multicolumn{7}{|l|}{ H.Pylori } \\
\hline Yes & 1193 & 246 & 34 & 0.413 & 0.566 & 1.236 \\
\hline No & & 947 & 212 & & & \\
\hline \multicolumn{7}{|l|}{ Alcohol } \\
\hline Yes & 1640 & 641 & 99 & 0.900 & 0.773 & 1.340 \\
\hline No & & 999 & 542 & & & \\
\hline \multicolumn{7}{|l|}{ Smoking } \\
\hline Yes & 1457 & 332 & 59 & 0.191 & 0.899 & 1.703 \\
\hline No & & 1325 & 273 & & & \\
\hline
\end{tabular}


Table 8 (continued)

\begin{tabular}{|c|c|c|c|c|c|c|}
\hline Variable & $\begin{array}{l}\text { Total no of } \\
\text { patients }\end{array}$ & No of patients & Complication & $p$ value & CI $(95 \%)$ & \\
\hline \multicolumn{7}{|l|}{ NSAIDS } \\
\hline Yes & 1704 & 125 & 21 & 0.776 & 0.659 & 1.749 \\
\hline No & & 1579 & 104 & & & \\
\hline \multicolumn{7}{|c|}{ Corticosteroids } \\
\hline Yes & 1704 & 110 & 22 & 0.226 & 0.830 & 2.196 \\
\hline No & & 1594 & 88 & & & \\
\hline \multicolumn{7}{|c|}{ Anticoagulants } \\
\hline Yes & 1704 & 121 & 26 & 0.083 & 9.49 & 2.355 \\
\hline No & & 1583 & 95 & & & \\
\hline \multicolumn{7}{|c|}{ Cholecystectomy } \\
\hline Yes & 1709 & 201 & 37 & 0.293 & 0.837 & 1.802 \\
\hline No & & 1508 & 164 & & & \\
\hline \multicolumn{7}{|c|}{ Revisional surgery } \\
\hline Yes & 1709 & 317 & 68 & 0.003 & 1.177 & 2.174 \\
\hline No & & 1392 & 249 & & & \\
\hline
\end{tabular}

Bold values indicate statistical analysis at $p<0.05$

$C I$ confidence interval, $B M I$ body mass index, NIDDM non-insulin depended diabetes mellitus, IDDM insulin depended diabetes mellitus, $O S A S$ obstructive sleep apnea, $A H I$ apnea hypopnea index, GERD gastroesophageal reflux disease $H$. Pylori helicobacter pylori, COPD chronic obstructive pulmonary disease, NSAIDS non-steroidal anti-inflammatory drugs

\section{References}

1. Buchwald H, (2013) Oien DM. Metabolic/bariatric surgery worldwide 2011. Obes Surg 23:427-436

2. Colquitt JL, Pickett K, Loveman E et al (2014) Surgery for weight loss in adults. Cochrane Database Syst Rev 8:CD003641

3. World Health Organisation. Obesity and Overweight. http:// www.who.int/mediacentre/factsheets/fs311/en/ August 2014; Accessed 14 Jan 2015

4. DeMaria EJ, Portenier D, Wolfe L (2007) Obesity surgery mortality risk score: proposal for a clinically useful score to predict mortality risk in patients undergoing gastric bypass. Surg Obes Relat Dis 3:134-140

5. Efthimiou E, Court O, Sampalis J et al (2009) Validation of obesity surgery mortality risk score in patients undergoing gastric bypass in a canadian center. Surg Obes Relat Dis 5:643-647

6. Sarela AI, Dexter SP, McMahon MJ (2011) Use of the obesity surgery mortality risk score to predict complications of laparoscopic bariatric surgery. Obes Surg 21:1698-1703

7. Geubbels N, de Brauw LM, Acherman YI et al (2014) The preceding surgeon factor in bariatric surgery: a positive influence on the learning curve of subsequent surgeons. Obes Surg doi:10.1007/s11695-014-1538-x

8. Dindo D, Demartines N, Clavien PA (2004) Classification of surgical complications: a new proposal with evaluation in a cohort of 6336 patients and results of a survey. Ann Surg 240:205-213

9. Zhang L, Tan WH, Chang R et al (2014) Perioperative risk and complications of revisional bariatric surgery compared to primary Roux-en-Y gastric bypass. Surg Endosc doi:10.1007/ s00464-014-3848-4

10. Goitein D, Raziel A, Szold A et al (2015) Assessment of perioperative complications following primary bariatric surgery according to the Clavien-Dindo classification: comparison of sleeve gastrectomy and Roux-Y gastric bypass. Surg Endosc doi:10.1007/s00464-015-4205-y
11. Anderin C, Gustafsson UO, Heijbel N et al (2015) Weight loss before bariatric surgery and postoperative complications: data from the Scandinavian Obesity Registry (SOReg). Ann Surg 261:909-913

12. Flum DR, Belle SH, King WC et al (2009) Perioperative safety in the longitudinal assessment of bariatric surgery. N Engl J Med 361:445-454

13. IFSO. IFSO: are you a candidate. http://www.ifso.com January 2015; Available from http://www.ifso.com Accessed 14 Jan 2015

14. Coblijn UK, Lagarde SM, de Castro SM et al (2014) Symptomatic marginal ulcer disease after Roux-en-Y gastric bypass: incidence, risk factors and management. Obes Surg doi:10.1007/ s11695-014-1482-9

15. Collins GS, Reitsma JB, Altman DG et al (2015) Transparent reporting of a multivariable prediction model for individual prognosis or diagnosis (TRIPOD): the TRIPOD statement. BMJ 350:g7594

16. Lorente L, Ramon JM, Vidal P et al (2014) Obesity surgery mortality risk score for the prediction of complications after laparoscopic bariatric surgery. Cir Esp 92:316-323

17. Gupta PK, Franck C, Miller WJ et al (2011) Development and validation of a bariatric surgery morbidity risk calculator using the prospective, multicenter NSQIP dataset. J Am Coll Surg 212:301-309

18. Stenberg E, Szabo E, Agren G et al (2014) Early complications after laparoscopic gastric bypass surgery: results from the Scandinavian Obesity Surgery Registry. Ann Surg 260:1040-1047

19. Finks JF, Kole KL, Yenumula PR et al (2011) Predicting risk for serious complications with bariatric surgery: results from the Michigan Bariatric Surgery Collaborative. Ann Surg 254:633-640

20. Birkmeyer JD, Finks JF, O'Reilly A et al (2013) Surgical skills and complication rates after bariatric surgery. N Engl J Med 369:1434-1442

21. Kristensen SD, Naver L, Jess P et al (2014) Effect of closure of the mesenteric defect during laparoscopic gastric bypass and prevention of internal hernia. Dan Med J 61:A4854 